\title{
Science-based, evidence-informed \& non-judgmental motivations to change maladaptive habits
}

\begin{abstract}
Background: Guilt, shame and stigma are the regular diet of the twelvestep/'anonymous' groups attended regularly as a supposed requirement of recovery. These groups tend to promote a dogmatic way of looking at and solving problematic habits that, for some people, can be in opposition to mastering their bad habits and promoting \& replacing them with good ones. Oftentimes, this dogmatism promotes a religious aspect of recovery that many find contrary to evidence- this aspect seems to require adopting a "higher power" that apparently does not have to be god, and regularly shaming oneself when the plan doesn't go exactly as expected. It is my hope that we can begin to abolish the notion of shame and stigma at one's maladaptive behaviorthis will require an honest look at whether religion and faith are a requirement for both living a balanced life and replacing bad habits with good habits.
\end{abstract}

Keywords: twelve-step, religion, shame, stigma, self-worth, higher power, maladaptive habi
Volume 5 Issue 6 - 2018

\author{
David Weidman \\ Tulane University/SMART Recovery, USA
}

\begin{abstract}
Correspondence: David Weidman, Tulane University/Smart recovery, 350 s Peck Dr Beverly Hills, CA 90212, USA, Tel 310
\end{abstract} 625-6058,Email waidmandavid@gmail.com

Received: October 4, 2018 | Published: November 19, 2018

\section{Introduction}

As the opioid epidemic continues to escalate exponentially and claim the lives of young and old, rich and poor, we find ourselves at a point in time when we have to ask some hard questions: Why isn't treatment helping more people? Why do people continue to overdose at an alarmingly increasing rate? What can we do to change this course? And why are there so few truly helpful options?

I have had the benefit of witnessing the growth of the epidemic firsthand, both as a substance user (my drug of choice until January 2010 was Oxycontin) and since as a facilitator and Regional Coordinator in Los Angeles for the organization I volunteer for, SMART Recoveryfounded in 1992 by Dr. Joseph Gerstein. SMART Recovery is agnostic as to what maladaptive behavior one is trying to change- our groups include all "addictions"- from drugs and alcohol to food, gambling and sex, and everything in between. ${ }^{1}$ I even found myself recently helping someone in the group whose only maladaptive habit was procrastination. And among SMART participants, the single greatest complaint I hear from those who had previously utilized twelve-step meetings is the higher power-based aspects of the "anonymous" groups. I have the added benefit of holding the only SMART Recovery meetings at a Hazelden Betty Ford Center [12-step based].

There is a growing trend among the people I speak to in the recovery community regarding the dependency in the field of addiction medicine on religion. This irrational belief- that somehow addiction to something, anything, is a moral failing to be fixed with guilt, shame and stigmatization- remains alive in our culture as if the year were 1957. We have made so much scientific progress in the years since, both in our understanding of the brain, as well as in human behaviors and their consequences, that it is truly stunning the number of new participants to SMART Recovery (Self Management \& Addiction Recovery Training) I meet who feel their maladaptive habits are based on morality- they are convinced that they are bad people.

This cultural phenomenon began in the 70's, when drug addict meant heroin abuser and the only way out of addiction was through a religious rebirth. Fast forward to the 1990's, when it became necessary to treat any addiction to a substance as a disease, which needed to be stamped out- quite similar to Lyndon Johnson's war on poverty?
Whenever Americans want to cover their eyes and bury their heads in the sand, they create a war. Take sides. Us against them. In this case, it was "addicts" and "alcoholics" against the "normal" drinkers $\&$ druggers. This opened the door for insurance companies to cover addiction treatment, since the medical community now had a disease to conquer, a fixable problem.

And where have these wars gotten us today? We are in the middle of one of the worst epidemics in human history, the opioid epidemic, taking the lives of approximately 175 of our fellow citizens per day. How we treat our fellow citizens matters, and it is my profound belief that we can come together in kindness and compassion, and begin to consider that what you refer to as an "addict" or an "alcoholic" is a real person; someone with feelings, desires- a full human being, with failings and successes abounding virtually every moment.

\section{This is the essence of the problem. As Alan watts so brilliantly pointed out in the early 70's:}

This feeling of being lonely and very temporary visitors in the universe is in flat contradiction to everything known about man (and all other living organisms) in the sciences. We do not "come into" this world; we come out of it, as leaves from a tree. As the ocean "waves," the universe "peoples." Every individual is an expression of the whole realm of nature, a unique action of the total universe. This fact is rarely, if ever, experienced by most individuals. Even those who know it to be true in theory do not sense or feel it, but continue to be aware of themselves as isolated "egos" inside bags of skin.

What we do habitually cannot be the only defining aspect of a human being- especially since many of our habits are based on irrational beliefs about our self worth. And this brings us full circle to the present. How we treat each other matters. With non-judgmental kindness and motivational techniques based on science and provable, repeatable outcomes, we help our participants move to changing, however they want. No faith, except in oneself, is required. And a willingness to master one's fears without believing that they can be "overcome". Kindness and inclusion will begin to change the way our society looks at maladaptive habits. As SMART's motto says, "Discover the Power of Change". 


\section{Funding details}

About funding agency.

\section{Conflict of interests}

Author declares that there is no conflict of interest.

\section{Acknowledgements}

None.

\section{References}

1. Smartrecovery.Org 